\title{
Pharmacological Treatment of Schizophrenia: Japanese Expert Consensus
}

\section{(ㄷ) (1) (우)}

\author{
Authors \\ Hitoshi Sakurai ${ }^{1,2}$, Norio Yasui-Furukori ${ }^{3}$, Takefumi Suzuki ${ }^{4}$, Hiroyuki Uchida², Hajime Baba5 ${ }^{5}$ Koichiro Watanabe ${ }^{6}$, \\ Ken Inada7, Yuka Sugawara Kikuchi ${ }^{8}$, Toshiaki Kikuchi², Asuka Katsuki ${ }^{9}$, Ikuko Kishida ${ }^{10,11}$, Masaki Kato ${ }^{12}$
}

\author{
Affiliations \\ 1 Department of Psychiatry, Massachusetts General \\ Hospital, Boston, MA, USA \\ 2 Department of Neuropsychiatry, Keio University School \\ of Medicine, Tokyo, Japan \\ 3 Department of Psychiatry, Dokkyo Medical University \\ School of Medicine, Tochigi, Japan \\ 4 Department of Neuropsychiatry, University of Yama- \\ nashi Faculty of Medicine, Yamanashi, Japan \\ 5 Department of Psychiatry \& Behavioral Science, Juntendo \\ University Graduate School of Medicine, Tokyo, Japan \\ 6 Department of Neuropsychiatry, Kyorin University \\ School of Medicine, Tokyo, Japan \\ 7 Department of Psychiatry, Tokyo Women's Medical \\ University School of Medicine, Tokyo Japan \\ 8 Department of Psychiatry, Akita University School of \\ Medicine, Akita, Japan \\ 9 Department of Psychiatry, University of Occupational \\ and Environmental Health, Fukuoka, Japan \\ 10 Fujisawa Hospital, Kanagawa, Japan \\ 11 Department of Psychiatry, Yokohama City University \\ School of Medicine, Kanagawa, Japan \\ 12 Department of Neuropsychiatry, Kansai Medical \\ University, Osaka, Japan
}

Key words antipsychotics, expert consensus, pharmacotherapy, schizophrenia

revised $\quad 19.11 .2020$

received $\quad 16.07 .2020$

accepted 22.11.2020

published online 12.1.2021

\author{
Bibliography \\ Pharmacopsychiatry 2021; 54: 60-67 \\ DOI 10.1055/a-1324-3517 \\ ISSN $\quad 0176-3679$ \\ (C) 2021. The Author(s). \\ This is an open access article published by Thieme under the terms of the \\ Creative Commons Attribution-NonDerivative-NonCommercial-License, \\ permitting copying and reproduction so long as the original work is given \\ appropriate credit. Contents may not be used for commecial purposes, or \\ adapted, remixed, transformed or built upon. (https://creativecommons.org/ \\ licenses/by-nc-nd/4.0/) \\ Georg Thieme Verlag KG, Rüdigerstraße 14, \\ 70469 Stuttgart, Germany
}

\section{Correspondence}

Masaki Kato, MD PhD

Department of Neuropsychiatry

Kansai Medical University

2-5-1 Shin-Machi Hirakata-Shi

Osaka

Japan

katom@takii.kmu.ac.jp

$\oplus$ Supplementary Material is available under https://doi.org/10.1055/a-1324-3517.

\section{ABSTRACT}

Introduction Conventional treatment guidelines of schizophrenia do not necessarily provide solutions on clinically important issues.

Methods A total of 141 certified psychiatrists of the Japanese Society of Clinical Neuropsychopharmacology evaluated treatment options regarding 19 clinically relevant situations in the treatment of schizophrenia with a 9-point scale (1 = "disagree" and 9 = "agree").

Results First-line antipsychotics varied depending on predominant symptoms: risperidone (mean \pm standard deviation score, $7.9 \pm 1.4$ ), olanzapine (7.5 \pm 1.6$)$, and aripiprazole (6.9 \pm 1.9 ) were more likely selected for positive symptoms; aripiprazole (7.6 \pm 1.6$)$ for negative symptoms; aripiprazole (7.3 \pm 1.9 ), olanzapine (7.2 \pm 1.9$)$, and quetiapine (6.9 \pm 1.9$)$ for depression and anxiety; and olanzapine (7.9 \pm 1.5$)$ and risperidone $(7.5 \pm 1.5)$ for excitement and aggression. While only aripiprazole was categorized as a first-line treatment for relapse prevention $(7.6 \pm 1.0)$ 
in patients without noticeable symptoms, aripiprazole (8.0 \pm 1.6$)$ and brexpiprazole $(6.9 \pm 2.3)$ were categorized as such for social integration. First-line treatments in patients who are vulnerable to extrapyramidal symptoms include quetiapine $(7.5 \pm 2.0)$ and aripiprazole $(6.9 \pm 2.1)$.
Discussion These clinical recommendations represent the expert consensus on the use of a particular antipsychotic medication for a particular situation, filling a current gap in the literature.

\section{Introduction}

Several treatment guidelines are published and widely used to improve the quality of care and treatment outcomes in schizophrenia. They describe treatment planning, benefits and harms of antipsychotic medications, and non-pharmacological approaches derived from evidence that is mainly based on randomized controlled trials (RCTs) and subsequent meta-analyses of RCTs [1-9]. Nevertheless, the strict inclusion/exclusion criteria of RCTs are considered to be a serious concern in terms of generalizability as the evidence may not necessarily address the situations encountered in daily clinical practice. Moreover, the guidelines fail to present solid treatment recommendations for clinical situations that past literature has not addressed. For instance, few of the currently available treatment guidelines suggest specific antipsychotic medications for predominantly negative symptoms and comorbid depressive, anxious, or obsessive-compulsive symptoms, while all of the guidelines indicate suitable antipsychotic doses and adverse effects of antipsychotics [1-9].

Opinions from field experts are considered useful to fill such a gap in the literature; their consensus reflecting actual clinical experience could develop recommendations for clinically challenging scenarios that have not been sufficiently investigated in RCTs to date.

The Japanese Society of Clinical Neuropsychopharmacology (JSCNP) is an academic society devoting substantial efforts to the improvement of clinical psychopharmacology for psychiatric disorders. The society has a board certification system in which its member psychiatrists are certified as specialists in this field, depending on their academic activities as well as scores on written examinations regarding professional expertise. There have been 277 psychiatrists certified as of November 2019. The society has recently published an expert consensus for depression [10] and has also developed one for bipolar disorder [11].

In this article, a consensus on choices for clinically relevant issues in the treatment of schizophrenia was established by the board-certified experts of the JSCNP. Recommended options were sought in the following 3 areas, all of which are critically important but still lack a solid evidence base in previous investigations: pharmacological strategy depending on clinical situations, choice of long-acting injectable antipsychotics, and discontinuation of pharmacotherapy.

\section{Methods}

\section{Study Design}

This survey was conducted from February 19, 2019 to April 25, 2019. After a thorough assessment of the currently available treat- ment guidelines for schizophrenia, the Medical Education Panel of the JSCNP, consisting of 13 academic experts, identified 19 clinical situations that had not been adequately addressed in the past literature. For each situation, a precisely described question and treatment choices were suggested by the panel. The certified psychiatrists of the JSCNP throughout the country were invited to participate in this survey by email. Those who agreed to participate were asked to evaluate the suggested treatment choices using a 9-point Likert scale (1 = "strongly disagree" and 9 = "strongly agree"). These clinical situations and treatment options are shown in Supplementary $>$ Table 1S. Experts were asked to choose a score of 9 for at least 1 choice if they would use at least one of the listed treatment choices. They were also asked to choose a score of 1 for all choices if they did not endorse any of the treatment choices listed. The survey took approximately 15 to $30 \mathrm{~min}$ to complete. Participation was voluntary and without any incentives. Respondents were asked to provide information on their age, gender, and work location.

\section{Analysis}

The following values were calculated for each treatment option; mean, standard deviation (SD), 95\% confidence interval (CI), and the number of responses within 1-3 (disagree), 4-6 (neutral), and 7-9 (agree). Pearson's chi-squared test was used to compare the frequencies of these responses (i.e., disagree, neutral, and agree) for each of the treatment choices. When the responses were evenly distributed across the 3 rating categories, as indicated by a p-value $\geq 0.05$, "no consensus" declaration was made regarding the situation. Treatment options with the lowest $95 \% \mathrm{Cl}$ value $\geq 6.5$ were regarded as "first-line treatments;" those with the lowest $95 \%$ $\mathrm{Cl}$ value $\geq 3.5$ were considered as "second-line treatments;" and the others were treated as "third-line treatments." Choices rated as 9 by more than $50 \%$ of the responders were defined as "treatments of choice." In general, the first-line treatment is usually appropriate as the initial treatment for a given situation [12]. The treatment of choice is a particularly strong first-line recommendation. The second-line treatment is a reasonable choice for patients who do not respond to or cannot tolerate the first-line treatment. The no consensus treatment is a controversial strategy. The thirdline treatment is usually inappropriate or used only when preferred alternatives are found to be ineffective [12]. Since expert consensuses based on the response to the questionnaires may be subject to biases, the panel discussed interpretation of the survey data and compared the results to the evidence currently available in the literature. 
- Table 1 Consensus on choice of antipsychotics depending on predominant symptoms.

\begin{tabular}{|c|c|c|c|c|}
\hline & Positive symptoms & Negative symptoms & Depression/anxiety & Excitement/aggression \\
\hline Aripiprazole & $1 \mathrm{st}$ & $1 \mathrm{st}$ & $1 \mathrm{st}$ & 2nd \\
\hline Olanzapine & 1st & 2nd & $1 \mathrm{st}$ & 1st \\
\hline Risperidone & 1st & 2nd & 2nd & $1 \mathrm{st}$ \\
\hline Quetiapine & 2nd & 2nd & $1 s t$ & 2nd \\
\hline Blonanserin & 2nd & 2nd & 2nd & 2nd \\
\hline Paliperidone & 2nd & 2nd & 2nd & 2nd \\
\hline Brexpiprazole & 2nd & 2nd & 2nd & 2nd \\
\hline Asenapine & 2nd & 2nd & 2nd & 2nd \\
\hline Clozapine & 2nd & 2nd & 2nd & 2nd \\
\hline Perospirone & 2nd & 2nd & 2nd & 2nd \\
\hline Haloperidol & 2nd & $3 r d$ & $3 r d$ & 2nd \\
\hline Zotepine & $3 r d$ & $3 r d$ & $3 r d$ & 2nd \\
\hline Chlorpromazine & $3 r d$ & $3 r d$ & $3 r d$ & 2nd \\
\hline Levomepromazine & $3 r d$ & $3 r d$ & $3 r d$ & 2nd \\
\hline Sulpiride & $3 r d$ & $3 r d$ & 2nd & $3 r d$ \\
\hline Fluphenazine & $3 r d$ & $3 r d$ & $3 r d$ & $3 r d$ \\
\hline Perphenazine & $3 r d$ & $3 r d$ & $3 r d$ & $3 r d$ \\
\hline
\end{tabular}

\section{Results}

\section{Participant characteristics}

Out of the 277 certified psychiatrists invited to participate, 141 completed the questionnaire (response rate: $50.9 \%$ ), securing a large enough number of respondents. Twenty-three respondents (16.3\%) were in their 30's, 47 (33.3\%) in their 40's, $43(30.5 \%)$ in their 50's, 26 (18.4\%) in their 60's, and 2 (1.4\%) in their 70's or older. The proportion of males was $90.8 \%$. Fifty-four respondents (38.3\%) were affiliated with university hospitals, 47 (33.3\%) with psychiatric hospitals, 15 (10.6\%) with community clinics, 11 (7.8\%) with general hospitals, and $14(9.9 \%)$ with other institutions such as government offices.

\section{Pharmacological strategy depending on clinical characteristics}

The choice of first-line antidepressants varied in accordance with predominant symptoms ( $\triangleright$ Table 1$)$. Risperidone (7.9 \pm 1.4 ), olanzapine $(7.5 \pm 1.6)$, and aripiprazole $(6.9 \pm 1.9)$ were highly selected for positive symptoms; aripiprazole $(7.6 \pm 1.6)$ was highly selected for negative symptoms; aripiprazole $(7.3 \pm 1.9)$, olanzapine (7.2 \pm 1.9$)$, and quetiapine $(6.9 \pm 1.9)$ were highly selected for depression and anxiety symptoms; and olanzapine (7.9 \pm 1.5$)$ and risperidone $(7.5 \pm 1.5)$ were highly selected for excitement and aggression. All other second-generation antipsychotics (SGAs) were categorized as second-line treatments for these 4 clinical features. On the other hand, first-generation antipsychotics (FGAs) were categorized as third-line treatments. However, haloperidol was categorized as a second-line treatment for positive symptoms $(5.5 \pm 2.6)$; sulpiride was categorized as a second-line treatment for depression and anxiety symptoms $(4.3 \pm 2.6)$; and zotepine $(6.3 \pm 2.4)$, levomepromazine $(5.8 \pm 2.6)$, haloperidol $(5.5 \pm 2.5)$, and chlorpromazine $(5.3 \pm 2.6)$ were categorized as second-line treatments for excitement and aggression.

The only first-line treatment for elderly patients was aripiprazole $(7.5 \pm 1.8)$ ( Table 2 ). While all other SGAs were categorized as second-line treatments for elderly patients, all FGAs were categorized as third-line treatments.

Aripiprazole was categorized as a first-line treatment for preventing relapse $(7.6 \pm 1.0)$ and the treatment of choice for social integration $(8.0 \pm 1.6)$ in a case without noticeable symptoms ( $\triangleright$ Table 2). Brexpiprazole was considered to be a second-line treatment for preventing relapse $(6.6 \pm 2.4)$ and a first-line treatment for social integration $(6.9 \pm 2.3)$. While all other SGAs were categorized as second-line treatments for both clinical situations-except for clozapine, which was categorized as no consensus-all FGAs were categorized as third-line treatments.

\section{Pharmacological treatment of clinical issues}

Quetiapine (7.5 \pm 2.0$)$ and aripiprazole $(6.9 \pm 2.1)$ were categorized as first-line treatments in a patient who has a high risk for extrapyramidal side effects ( $\triangleright$ Table 2 ). They were followed by second-line SGAs, including olanzapine $(6.6 \pm 2.0)$, brexpiprazole $(6.6 \pm 2.4)$, and clozapine $(6.3 \pm 2.7)$. All FGAs were categorized as third-line treatments.

No choice was categorized as a first-line treatment for adjunctive use with antipsychotic medication for excitement during acute treatment. Notably, risperidone was categorized as a second-line treatment $(6.1 \pm 2.6)$ ( $\triangleright$ Table 3). On the other hand, risperidone was the only first-line treatment for pro re nata (PRN) in temporal excitement or agitation $(6.9 \pm 2.4)$ ( $\triangleright$ Table 3$)$. Olanzapine $(6.8 \pm 2.5$ and $6.6 \pm 2.5$, respectively), lorazepam $(5.9 \pm 2.6$ and $6.2 \pm 2.6$, respectively), quetiapine $(6.2 \pm 2.6$ and $6.1 \pm 2.7$, respectively), and levomepromazine $(5.8 \pm 2.8$ and $5.5 \pm 2.9$, respectively) were considered to be second-line treatments both for concomitant medi- 
- Table 2 Consensus on choice of antipsychotics depending on clinical situations.

\begin{tabular}{|c|c|c|c|c|}
\hline & Elderly & Relapse prevention & Social integration & Vulnerability to EPS \\
\hline Aripiprazole & 1st & $1 \mathrm{st}$ & Best & $1 \mathrm{st}$ \\
\hline Brexpiprazole & 2nd & 2nd & 1st & 2nd \\
\hline Quetiapine & 2nd & 2nd & 2nd & 1st \\
\hline Risperidone & 2nd & 2nd & 2nd & 2nd \\
\hline Paliperidone & 2nd & 2nd & 2nd & 2nd \\
\hline Asenapine & 2nd & 2nd & 2nd & 2nd \\
\hline Olanzapine & 2nd & 2nd & 2nd & 2nd \\
\hline Perospirone & 2nd & 2nd & 2nd & No consensus \\
\hline Blonanserin & 2nd & 2nd & 2nd & No consensus \\
\hline Clozapine & 2nd & No consensus & No consensus & 2nd \\
\hline Haloperidol & $3 r d$ & $3 r d$ & $3 r d$ & $3 r d$ \\
\hline Zotepine & $3 r d$ & $3 r d$ & $3 r d$ & $3 r d$ \\
\hline Chlorpromazine & $3 r d$ & $3 r d$ & $3 r d$ & $3 r d$ \\
\hline Levomepromazine & $3 r d$ & $3 r d$ & $3 r d$ & $3 r d$ \\
\hline Fluphenazine & $3 r d$ & $3 r d$ & $3 r d$ & $3 r d$ \\
\hline Perphenazine & $3 r d$ & $3 r d$ & $3 r d$ & $3 r d$ \\
\hline Sulpiride & $3 r d$ & $3 r d$ & $3 r d$ & $3 r d$ \\
\hline
\end{tabular}

cations in persistent excitement and for PRNs in temporal excitement or agitation. While zotepine, electroconvulsive therapy (ECT), sodium valproate, paliperidone, and haloperidol were categorized as second-line treatments for concomitant use and not for PRNs, diazepam was categorized as a second-line treatment for PRN and not for concomitant use.

No choice was categorized as a first-line treatment for concomitant use with antipsychotic medication in depression and anxiety ( Table 3). Some choices, including quetiapine $(5.5 \pm 2.7)$, were considered to be second-line treatments. There was also no choice categorized as a first-line treatment for concomitant use with antipsychotic medication for obsessive-compulsive symptoms ( $\triangleright$ Table 3). Second-line treatments included selective serotonin reuptake inhibitors (SSRI) $(6.1 \pm 2.8)$ and aripiprazole $(4.1 \pm 2.7)$.

Regarding the therapeutic strategy for treatment-resistant schizophrenia, switching to clozapine was categorized as the treatment of choice $(7.7 \pm 2.3)$ and ECT as a first-line treatment $(7.5 \pm 2.1)$ (Supplementary $\triangleright$ Table 1S). They were followed by second-line treatments including switching to another antipsychotic medication $(6.7 \pm 2.2)$, adding another antipsychotic medication $(6.1 \pm 2.3)$, and adding a mood stabilizer $(5.6 \pm 2.4)$.

\section{Choice of long-acting injectable antipsychotics}

The leading reasons for introducing a long-acting injectable (LAI) antipsychotic were patient's request $(7.8 \pm 2.0$, treatment of choice), poor medication adherence (7.6 \pm 1.9$)$, and repeated recurrences $(7.4 \pm 2.1)$ (Supplementary — Table 1S).

Paliperidone palmitate was categorized as a first-line treatment for positive symptoms $(7.2 \pm 2.3)$, followed by aripiprazole LAI $(6.6 \pm 2.4)$ and risperidone $\operatorname{LAl}(6.5 \pm 2.5)$ as second-line treatments (Supplementary $>$ Table 1S). On the other hand, aripiprazole LAI was considered to be a first-line treatment for negative symptoms (7.4 \pm 2.5$)$, followed by paliperidone palmitate $(5.9 \pm 2.5)$.

\section{Discontinuation of pharmacotherapy}

Various factors were taken into consideration when planning a decrease in antipsychotic medication: presence/degree of side effects $(8.0 \pm 1.2)$, duration of clinical stabilization (7.6 \pm 1.8$)$, remaining symptoms (7.6 \pm 1.6$)$, amount of current medicine (7.5 \pm 1.6$)$, patient's understanding of relapse prevention (7.5 \pm 1.8$)$, current social adaptation $(7.5 \pm 1.6)$, symptom severity when deteriorated $(7.4 \pm 1.7)$, patient's understanding of early signs of relapse (7.4 \pm 1.6$)$, number of past episodes $(7.3 \pm 1.9)$, past treatment response (7.3 \pm 1.7$)$, understanding of illness (7.3 \pm 1.9$)$, and wish to have children $(7.1 \pm 1.8)$ (Supplementary $>$ Table 1S). On the other hand, the following factors were considered as first-line choices when planning the termination of antipsychotic medication: duration of clinical stabilization $(7.1 \pm 2.4)$, remaining symptoms $(7.1 \pm 2.5)$, and patient's understanding of relapse prevention $(7.1 \pm 2.4)$.

Regarding the duration of concomitant use of benzodiazepine anxiolytics, the only first-line option was PRN (7.2 \pm 2.3$)$ (Supplementary - Table 1S). The following choice was "within 1 month" as the only second-line option $(6.3 \pm 2.7)$, indicating a shorter duration of concomitant use received a higher agreement level.

\section{Discussion}

In the present study, treatment options for clinical situations in real-world patients with schizophrenia, which had not been sufficiently addressed in the conventional treatment guidelines, were evaluated by the experts in Japan with a simple statistical methodology. On the whole, SGAs were recommended as first-line or second-line treatments in almost all available situations, regardless of oral or LAI formulations. More notably, aripiprazole was highly endorsed in several clinical scenarios because of its relatively benign risk and benefit ratio. These recommendations emphasize the considera- 
- Table 3 Consensus on concomitant or PRN medication with an antipsychotic depending on clinical situations.

\begin{tabular}{|c|c|c|c|c|}
\hline & $\begin{array}{l}\text { Reduction of } \\
\text { excitement }\end{array}$ & $\begin{array}{l}\text { PRN for excitement/ } \\
\text { agitation }\end{array}$ & $\begin{array}{l}\text { Depression/ } \\
\text { anxiety }\end{array}$ & $\begin{array}{l}\text { Obsessive compulsive } \\
\text { symptoms }\end{array}$ \\
\hline Risperidone & 2nd & $1 \mathrm{st}$ & $3 r d$ & $3 r d$ \\
\hline Quetiapine & 2nd & 2nd & 2nd & $3 r d$ \\
\hline Olanzapine & 2nd & 2nd & No consensus & $3 r d$ \\
\hline Levomepromazine & 2nd & 2nd & n.a. & n.a. \\
\hline Lorazepam & 2nd & 2nd & n.a. & n.a. \\
\hline Diazepam & $3 r d$ & 2nd & n.a. & n.a. \\
\hline Zotepine & 2nd & No consensus & n.a. & n.a. \\
\hline ECT & 2nd & n.a. & n.a. & n.a. \\
\hline Sodium valproate & 2nd & $3 r d$ & n.a. & n.a. \\
\hline Paliperidone & 2nd & $3 r d$ & n.a. & n.a. \\
\hline Haloperidol & 2nd & $3 r d$ & n.a. & n.a. \\
\hline Aripiprazole & $3 r d$ & $3 r d$ & No consensus & 2nd \\
\hline SSRI & n.a. & n.a. & No consensus & 2nd \\
\hline SNRI & n.a. & n.a. & 2nd & $3 r d$ \\
\hline Lithium & n.a. & n.a. & 2nd & $3 r d$ \\
\hline Chlorpromazine & No consensus & No consensus & n.a. & n.a. \\
\hline Asenapine & No consensus & No consensus & $3 r d$ & $3 r d$ \\
\hline Brexpiprazole & $3 r d$ & $3 r d$ & No consensus & $3 r d$ \\
\hline Sulpiride & $3 r d$ & $3 r d$ & $3 r d$ & $3 r d$ \\
\hline Blonanserin & $3 r d$ & $3 r d$ & n.a. & n.a. \\
\hline Perospirone & $3 r d$ & $3 r d$ & n.a. & n.a. \\
\hline Sultopride & $3 r d$ & $3 r d$ & n.a. & n.a. \\
\hline Fluphenazine & $3 r d$ & $3 r d$ & n.a. & n.a. \\
\hline Perphenazine & $3 r d$ & $3 r d$ & n.a. & n.a. \\
\hline Benzodiazepine & n.a. & n.a. & No consensus & $3 r d$ \\
\hline Mirtazapine & n.a. & n.a. & No consensus & $3 r d$ \\
\hline TCA & n.a. & n.a. & $3 r d$ & $3 r d$ \\
\hline $\mathrm{TeCA}$ & n.a. & n.a. & $3 r d$ & $3 r d$ \\
\hline Trazodone & n.a. & n.a. & $3 r d$ & $3 r d$ \\
\hline
\end{tabular}

tion of each patient's needs and symptomatology to individually devise pharmacotherapy in schizophrenia.

According to these expert consensus recommendations, all oral SGAs were categorized as first-line or second-line treatments in the 8 clinical scenarios (i.e., predominant positive symptoms, predominant negative symptoms, predominant depression and anxiety, predominant excitement and aggression, elderly patients, relapse prevention, social integration, and a high risk for extrapyramidal symptoms [EPS]), except for no consensus regarding clozapine for relapse prevention and social integration, as well as perospirone and blonanserin (Japanese antipsychotics) for a high risk for EPS. On the other hand, oral FGAs were categorized as third-line treatments in all of the scenarios, except for second-line treatments of haloperidol for positive symptoms, sulpiride for depression and anxiety, and zotepine, levomepromazine, haloperidol, and chlorpromazine for excitement and aggression. Similarly, while paliperidone and aripiprazole LAls were highly endorsed in both cases with predominant positive and negative symptoms, haloperidol and flu- phenazine LAls were not. The effectiveness and safety between FGAs and SGAs has been debated for decades. In meta-analyses published in 2010 [13] and 2013 [14], there were no differences between oral FGAs and SGAs in symptom-scale reduction in patients with early and first-episode psychosis. However, oral SGAs provided better outcomes than oral FGAs in terms of negative symptoms, depression, global cognition, long-term remission, relapse rate, and short-term and long-term all-cause discontinuation rates [14]. Furthermore, whereas oral SGAs were associated with more weight gain and a larger total cholesterol increase than oral FGAs, SGAs as a class had a lower incidence of EPS and akathisia, and less adjunctive anticholinergic and benzodiazepine use [14]. Taking into account such similar effectiveness on total symptom reduction, better outcomes in non-psychotic symptoms, and lower incidence of EPS in comparison with FGAs, SGAs were highly endorsed in several clinical settings. Likewise, while there was no difference in time to relapse in patients treated with FGA and SGA LAls according to a retrospective chart review [15], fluphenazine and 
haloperidol LAls, but not risperidone and paliperidone LAls, were associated with more EPS than placebo in a network meta-analysis [16]. The experts who participated in the present study endorsed SGAs at all times for the treatment of schizophrenia, probably in light of their higher short-term and long-term efficacy and lower risk of movement disorders in comparison with FGAs. In contrast to oral antipsychotics, there have been only a few reports that compared the efficacy and safety among the currently available LAls [17]. LAls are at least as effective as oral antipsychotics for schizophrenia [18].

One of the unique features of this expert consensus is that recommendations for the choices of medications in specific clinical features or symptoms are clearly stated. Many of them are consistent with the recommendations of previous treatment guidelines or findings of meta-analyses, but those regarding aripiprazole are somewhat different. For example, risperidone, olanzapine, and aripiprazole were categorized as first-line treatments for positive symptoms in the present study. While risperidone and olanzapine as well as clozapine improved positive symptoms to a greater extent than many other drugs according to a recent network metaanalysis of 402 RCTs on the efficacy and safety of 32 antipsychotics for several symptoms during acute treatment for adults with multi-episode schizophrenia, aripiprazole was ranked around the middle [19]. Similarly, aripiprazole, categorized as the only first-line treatment for negative symptoms, was again ranked around the middle in the network meta-analysis [19]. Instead, clozapine, amisulpride, and olanzapine improved negative symptoms more than many other antipsychotics in the network meta-analysis. Concerning depressive and anxious symptoms in schizophrenia, aripiprazole, olanzapine, and quetiapine were preferable possibly because of their antidepressant effects [20]. In fact, the network meta-analysis gave a higher rank to olanzapine and aripiprazole for depressive symptoms followed by sulpiride, clozapine, and amisulpride [19], and the World Federation of Societies of Biological Psychiatry Guideline also referred to the effectiveness of quetiapine in reducing depressive symptoms [3]. Olanzapine and risperidone were preferred for excitement and aggression in the present study, which is consistent with the guideline from the Scottish Intercollegiate Guidelines Network that suggested amisulpride, olanzapine, and risperidone for an acute exacerbation [9]. For comparison, the guidelines from the British Association for Psychopharmacology [6] and the Harvard South Shore Program [7] recommended clozapine for persistent hostility and violent behavior even if the patient was not treatment-resistant. Aripiprazole was categorized as first-line treatment for elderly patients, even though there is no RCT of aripiprazole in geriatric schizophrenia specifically [21]. In a meta-analysis of 18 RCTs for antipsychotic treatment in elderly patients with schizophrenia, olanzapine was more efficacious for overall symptoms compared to haloperidol [21]. Aripiprazole was preferred in preventing relapse in patients without noticeable symptoms, while the guideline from the Scottish Intercollegiate Guidelines Network suggested amisulpride, olanzapine, and risperidone for maintenance treatment [9]. Aripiprazole and brexpiprazole, which were endorsed for social integration in the present study, were ranked around the middle in improving social functioning in the network meta-analysis [19]. Alternatively, thioridazine, olanzapine, and paliperidone improved social functioning more than other antipsychotics in the network meta-analysis [19]. Quetiapine and aripiprazole were categorized as the first-line treatments for those who had a high risk of EPS. According to the network meta-analysis, quetiapine was less likely associated with akathisia and a need for anti-Parkinson medication, while aripiprazole was ranked lower for these respects [19].

Briefly, aripiprazole has not always been highly valued for some specific clinical features or symptoms in the previous treatment guidelines and network meta-analysis, whereas it was endorsed as a first-line treatment for many instances in our study. The probable explanation of this broad recommendation is a relatively benign risk and benefit ratio of aripiprazole, which appears to reflect the "above all, do no harm" principle. In fact, aripiprazole is associated with a lower risk of weight gain, metabolic syndrome, sedation, anticholinergic symptoms, prolactin elevation, QTc prolongation, and EPS compared to other antipsychotics [19, 22, 23]. However, expert consensus only has a much lower level of evidence in comparison with DBRCTs and meta-analyses [24]. In the most recent headto-head RCT of 144 patients with schizophrenia-spectrum disorders, amisulpride was more efficacious than aripiprazole or olanzapine for reducing symptom severity [25]. Moreover, aripiprazole ranks low in the network meta-analysis of 32 antipsychotic drugs [19]. Further, in a meta-analysis of 352 RCTs with a total of 84,988 patients with mixed diagnoses, aripiprazole showed a higher mortality rate than placebo [26]. Taken together, the potential gap between the expert consensus and higher level evidence should be appreciated. Lastly, it is fair to state that some authors of this article had a conflict of interest with Otsuka Pharmaceutical although the company was not at all involved in this work.

Concerning rescue medications for transient excitement or agitation, only risperidone was categorized as a first-line treatment possibly because of its rapid-action and sedative effects [27]. There were no first-line co-treatment psychotropics in persistent excitement, depressive and anxious symptoms, and obsessive-compulsive symptoms, which likely reflect the physicians' struggle to manage these challenging symptoms. In fact, there has been only equivocal evidence to base the choice of PRN for psychiatric patients [28]. While some treatment guidelines suggested that adjunctive antipsychotics [6], benzodiazepines [1, 5, 6], lithium [6], valproate $[6,9]$, carbamazepine [6, 9], and promethazine [9] might have some benefit for persistent agitation, aggression, or excitement and that co-prescribing antidepressants $[3,5,6]$, benzodiazepines [5], lithium [3,4], sodium valproate $[3,4,6]$, and carbamazepine [3] might do so for depressive symptoms, the evidence is still inconsistent and uncertain $[1,6]$. Due to a very limited number of controlled trials, the treatment of anxious symptoms [4, 9] and obsessive-compulsive symptoms [2] in schizophrenia may be guided by relevant guidelines on anxiety disorders and obsessive-compulsive disorder. Obsessive-compulsive symptoms in schizophrenia may be treated with antipsychotics while they may also contrarily cause them de novo [29]. Given that these symptoms are often observed in clinical practice, further research is necessary.

Multiple factors were to be considered in planning dose reduction of antipsychotics. The 3 factors of duration of clinical stabilization, remaining symptoms, and patient's understanding of relapse prevention, were categorized as first-line considerations when antipsychotic discontinuation was to be planned. Due to 
many problematic adverse events, it was recommended that benzodiazepines be prescribed as briefly as possible when used adjunctively in the treatment of schizophrenia, although longer-term use is frequent in the community [30].

This study should be interpreted with several limitations in mind. First, an expert consensus is considered as a lower level of evidence. However, many clinical questions have not been scientifically addressed in RCTs, which calls for consensus-based recommendations to fill the gap. Second, the questionnaire may not have included sufficient information from respondents in choosing appropriate treatment choices. The heterogeneity of patients should be taken into consideration when the recommendations in this guideline are translated into clinical practice. Third, the generalizability of this study may be limited, considering that all the participating experts were Japanese certified psychiatrists, and certain prescription habits among the Japanese experts may exist. In particular, there are more regulations concerning clozapine use in Japan than in other countries, which is likely to affect recommendations for clozapine [31, 32]. For example, in Japan, clozapine can only be initiated during an inpatient stay and weekly hematological monitoring is mandatory for the first 26 weeks, followed by biweekly blood tests thereafter. Also, some of the medications listed in the questionnaire are not available outside of Japan and vice versa (e.g., amisulpride, ziprasidone, and iloperidone have not been approved in Japan while perospirone and blonanserin are not widely available outside Asia). Finally, our categorization into 3 classes (i.e., 1-3 [disagree], 4-6 [neutral], and 7-9 [agree]) and our methods of analysis are somewhat arbitrary.

In conclusion, Japanese experts selected the optimal pharmacological treatment for schizophrenia based on patients' clinical characteristics and drug profiles. SGAs, especially aripiprazole, are highly endorsed for many of the clinical situations. Although these recommendations need to be supported by further clinical trials, the consensus-based recommendations may be useful to discuss therapeutic strategies in clinically challenging situations for which the evidence to date is limited.

\section{Conflicts of Interest}

Dr. Sakurai reports grants from the Japanese Society of Clinical Neuropsychopharmacology and the Uehara Memorial Foundation, and personal fees from Dainippon-Sumitomo Pharma, Otsuka Pharmaceutical, Meiji-Seika Pharma, Eli Lilly, Tanabe Mitsubishi Pharma, and Yoshitomi Yakuhin, outside the submitted work. Dr. Norio Yasui-Furukori has been a speaker for Dainippon-Sumitomo Pharmaceutical, Mochida Pharmaceutical, Otsuka Pharmaceutical, and MSD for other studies within the past 3 years. The funders had no role in the study design, data collection and analysis, the decision to publish, or the preparation of the manuscript. Dr. Suzuki has received manuscript or speaker's fees from Astellas, Dainippon Sumitomo Pharma, Eisai, Eli Lilly, Elsevier Japan, Janssen Pharmaceuticals, Kyowa Yakuhin, Meiji Seika Pharma, Mitsubishi Tanabe Pharma, MSD, Novartis, Otsuka Pharmaceutical, Shionogi, Shire, Tsumura, Wiley Japan, and Yoshitomi Yakuhin, and research grants from Eisai, Mochida Pharmaceutical, Meiji Seika Pharma, and Shionogi. Dr. Uchida has received grants from Eisai, Otsuka Pharmaceutical, Dainippon-Sumitomo Pharma, and Meiji-Seika Pharmaceutical; speaker's honoraria from Otsuka Pharmaceutical, Eli Lilly, Yoshitomi Yakuhin, Dainippon-Sumitomo Pharma, Meiji-Seika Pharma, and MSD; and advisory panel payments from Dainippon-Sumitomo Pharma with- in the past 3 years. Dr. Baba reports grants from Novartis, and personal fees from MSD, Otsuka, Dainippon Sumitomo, Meiji Seika, Eli Lilly, Yoshitomi, Janssen, Kyowa, Mitsubishi Tanabe, Ono, Pfizer, and Takeda, outside the submitted work. Dr. Watanabe has received manuscript fees or speaker's honoraria from Daiichi Sankyo, Eisai, Eli Lilly, GlaxoSmithKline, Janssen Pharmaceutical, Kyowa Pharmaceutical, Meiji Seika Pharma, Mitsubishi Tanabe Pharma, MSD, Otsuka Pharmaceutical, Pfizer, Shionogi, Sumitomo Dainippon Pharma, Takeda Pharmaceutical, and Yoshitomi and research/grant support from Astellas Pharma, Daiichi Sankyo, Eisai, MSD, Mitsubishi Tanabe Pharma, Meiji Seika Pharma, Otsuka Pharmaceutical, Pfizer, Shionogi, and Sumitomo Dainippon Pharma. Dr. Watanabe is a consultant of Eisai, Eli Lilly, Kyowa Pharmaceutical, Otsuka Pharmaceutical, Pfizer, Sumitomo Dainippon Pharma, Taisho Toyama Pharmaceutical, and Takeda Pharmaceutical. Dr. Inada reports personal fees from Dainippon Sumitomo Pharma, Eisai, Eli Lilly Japan, Janssen Pharmaceutical, Meiji-Seika Pharmaceutical, Mochida, Novartis, Otsuka Pharmaceutical, Shionogi, Tanabe-Mitsubishi Pharma, and Yoshitomi Yakuhin, and grants and personal fees from MSD, outside the submitted work. Dr. Yuka Sugawara Kikuchi has nothing to disclose. Dr. Toshiaki Kikuchi reports personal fees from Otsuka Pharmaceutical, Dainippon-Sumitomo, Eli Lilly, Mochida, Meiji-Seika, Yoshitomi-Yakuhin, Takeda, MSD, and Pfizer, outside the submitted work. Dr. Katsuki reports personal fees from Dainippon Sumitomo Pharma. Dr. Kishida has nothing to disclose. Dr. Kato has received grant funding from the Japan Society for the Promotion of Science, SENSHIN Medical Research Foundation, and Japan Research Foundation for Clinical Pharmacology, and speaker's honoraria from Sumitomo Dainippon Pharma, Otsuka, Meiji-Seika Pharma, Eli Lilly, MSD K.K., GlaxoSmithKline, Pfizer, Janssen Pharmaceutical, Shionogi, Mitsubishi Tanabe Pharma, Takeda Pharmaceutical, Lundbeck, and Ono Pharmaceutical.

\section{References}

[1] Hasan A, Falkai P, Wobrock T et al. World Federation of Societies of Biological Psychiatry (WFSBP) Guidelines for Biological Treatment of Schizophrenia, part 1: Update 2012 on the acute treatment of schizophrenia and the management of treatment resistance. World J Biol Psychiatry 2012; 13: 318-378

[2] Hasan A, Falkai P, Wobrock T et al. World Federation of Societies of Biological Psychiatry (WFSBP) Guidelines for Biological Treatment of Schizophrenia, part 2: update 2012 on the long-term treatment of schizophrenia and management of antipsychotic-induced side effects. World J Biol Psychiatry 2013; 14: 2-44

[3] Hasan A, Falkai P, Wobrock T et al. World Federation of Societies of Biological Psychiatry (WFSBP) Guidelines for Biological Treatment of Schizophrenia, part 3: update 2015 management of special circumstances: depression, suicidality, substance use disorders and pregnancy and lactation. World J Biol Psychiatry 2015; 16: 142-170

[4] Galletly C, Castle D, Dark F et al. Royal Australian and New Zealand College of Psychiatrists clinical practice guidelines for the management of schizophrenia and related disorders. Aust NZ J Psychiatry 2016; 50: 410-472

[5] Buchanan RW, Kreyenbuhl J, Kelly DL et al. The 2009 schizophrenia PORT psychopharmacological treatment recommendations and summary statements. Schizophr Bull 2010: 71-93

[6] Barnes TR, Drake R, Paton C et al. Evidence-based guidelines for the pharmacological treatment of schizophrenia: updated recommendations from the British Association for Psychopharmacology. J Psychopharmacol Oxf Engl 2020; 34: 3-78 
[7] Osser DN, Roudsari M], Manschreck T. The psychopharmacology algorithm project at the Harvard South Shore Program: An update on schizophrenia. Harv Rev Psychiatry 2013; 21: 18-40

[8] National Collaborating Centre for Mental Health (UK). Psychosis and Schizophrenia in Adults: Treatment and Management: Updated Edition 2014. 2014; http://www.ncbi.nlm.nih.gov/books/NBK248060/

[9] Scottish Intercollegiate Guidelines Network. Management of schizophrenia. 2013; SIGN 131: https://www.sign.ac.uk

[10] Sakurai H, Uchida H, Kato M et al. Pharmacological management of depression: Japanese expert consensus. J Affect Disord 2020; 266: 626-632

[11] Sakurai H, Kato M, Yasui-Furukori N et al. Pharmacological management of bipolar disorder: Japanese expert consensus. Bipolar Disord 2020; in press. doi: $10.1111 /$ bdi. 12959

[12] Allen MH, Currier GW, Hughes DH et al. Treatment of behavioral emergencies: a summary of the expert consensus guidelines. J Psychiatr Pract 2003; 9: 16-38

[13] Crossley NA, Constante M, McGuire P et al. Efficacy of atypical v. typical antipsychotics in the treatment of early psychosis: Meta-analysis. Br J Psychiatry. J Ment Sci 2010; 196: 434-439

[14] Zhang J-P, Gallego JA, Robinson DG et al. Efficacy and safety of individual second-generation vs. first-generation antipsychotics in first-episode psychosis: A systematic review and meta-analysis. Int J Neuropsychopharmacol 2013; 16: 1205-1218

[15] Stone JM, Roux S, Taylor D et al. First-generation versus second-generation long-acting injectable antipsychotic drugs and time to relapse. Ther Adv Psychopharmacol 2018; 8: 333-336

[16] Zhao Y], Lin L, Teng M et al. Long-term antipsychotic treatment in schizophrenia: Systematic review and network meta-analysis of randomised controlled trials. BJPsych Open 2016; 2: 59-66

[17] Naber D, Hansen K, Forray C et al. Qualify: A randomized head-to-head study of aripiprazole once-monthly and paliperidone palmitate in the treatment of schizophrenia. Schizophr Res 2015; 168: 498-504

[18] Suzuki T. A further consideration on long-acting injectable versus oral antipsychotics in the treatment of schizophrenia: A narrative review and critical appraisal. Expert Opin Drug Deliv 2016; 13: 253-264

[19] Huhn M, Nikolakopoulou A, Schneider-Thoma J et al. Comparative efficacy and tolerability of 32 oral antipsychotics for the acute treatment of adults with multi-episode schizophrenia: A systematic review and network meta-analysis. Lancet Lond Engl 2019; 394: 939-951

[20] Wang S-M, Han C, Lee S-J et al. Second generation antipsychotics in the treatment of major depressive disorder: an update. Chonnam Med J. 2016; 52: 159-172
[21] Krause M, Huhn M, Schneider-Thoma ] et al. Antipsychotic drugs for elderly patients with schizophrenia: A systematic review and meta-analysis. Eur Neuropsychopharmacol 2018; 28: 1360-1370

[22] Khanna P, Suo T, Komossa K et al. Aripiprazole versus other atypical antipsychotics for schizophrenia. Cochrane Database Syst Rev 2014; 1: CD006569

[23] Solmi M, Murru A, Pacchiarotti I et al. Safety, tolerability, and risks associated with first- and second-generation antipsychotics: A state-of-the-art clinical review. Ther Clin Risk Manag 2017; 13: 757-777

[24] Sackett DL, Straus SE, Richardson WS et al. Evidence-based medicine: How to practice and teach EBM. 2nd ed. Edinburgh: Churchill Livingstone; 2000

[25] Johnsen E, Kroken RA, Løberg E et al. Amisulpride, aripiprazole, and olanzapine in patients with schizophrenia-spectrum disorders (BeSt InTro): A pragmatic, rater-blind, semi-randomised trial. Lancet. Psychiatry 2020; 7: 945-954

[26] Schneider-Thoma J, Efthimiou O, Huhn $\mathrm{M}$ et al. Second-generation antipsychotic drugs and short-term mortality: A systematic review and meta-analysis of placebo-controlled randomised controlled trials. Lancet Psychiatry 2018; 5: 653-663

[27] Kelleher JP, Centorrino F, Albert M] et al. Advances in atypical antipsychotics for the treatment of schizophrenia: New formulations and new agents. CNS Drugs 2002; 16: 249-261

[28] Yoshida K, Suzuki T, Uchida H et al. Absence of evidence that the pro re nata regimen confers benefit: a review of the studies. Int Clin Psychopharmacol 2013; 28: 228-237

[29] Fonseka TM, Richter MA, Müller DJ. Second generation antipsychoticinduced obsessive-compulsive symptoms in schizophrenia: a review of the experimental literature. Curr Psychiatry Rep 2014; 16: 510

[30] Panes A, Pariente A, Bénard-Laribière A et al. Use of benzodiazepines and z-drugs not compliant with guidelines and associated factors: A population-based study. Eur Arch Psychiatry Clin Neurosci 2020; 270: 3-10

[31] Nielsen J, Young C, Ifteni P et al. Worldwide differences in regulations of clozapine use. CNS Drugs 2016; 30: 149-161

[32] Yoshida K, Suzuki T, Uchida H. Psychopharmacology in Japan: An overview and recent topics. J Clin Psychopharmacol 2018; 38 : 415-419 